KANSAS JOURNAL of MEDICINE

\section{Glioblastoma and Increased Survival with Longer Chemotherapy Duration}

Dory Abou Jaoude, M.D., MSCR ${ }^{1}$, Joseph A. Moore, M.D. ${ }^{2}$, Matthew B. Moore, B.A. ${ }^{3}$, Philip Twumasi-Ankrah, Ph.D., Elizabeth Ablah, Ph.D., MPH', Dennis F. Moore, Jr., M.D., FACP 5,6

${ }^{1}$ Ascension Via Christi, Wichita, KS

${ }^{2}$ University of Texas Southwestern Medical Center, Dallas, TX

${ }^{3}$ Beth Isreal Deaconess Medical Center, Boston, MA

${ }^{4}$ Astra Zeneca Pharmaceuticals, Gaithersburg, MD

${ }^{5}$ University of Kansas School of Medicine-Wichita

${ }^{6}$ Cancer Center of Kansas, Wichita, KS

Received June 5, 2018; Accepted for publication Feb. 20, 2019; Published online Aug. 21, 2019

\begin{abstract}
Introduction. The five-year survival rate for patients with glioblastoma (GBM) is low at approximately $4.7 \%$. Radiotherapy plus concomitant and adjuvant temozolomide (TMZ) remains the standard of care. The optimal duration of therapy with TMZ is unknown. This study sought to evaluate the survival benefit of two years of treatment.
\end{abstract}

Methods. This was a retrospective chart review of all patients diagnosed with GBM and treated with TMZ for up to two years between January 1, 2002 and December 31, 2011. The Kaplan-Meier method with log-rank test was used to estimate the progression-free survival (PFS) and the overall survival (OS). The results were compared to historical controls and data from previous clinical trials of patients treated up to one year.

Results. Data from 56 patients with confirmed GBM were evaluated. The OS probability was $54 \%(\mathrm{SE}=0.068)$ at one year, $28.3 \%$ $(\mathrm{SE}=0.064)$ at two years, $17.8 \%(\mathrm{SE}=0.059)$ at three years, and $4 \%(\mathrm{SE}=0.041)$ at five years. Seven patients $(12.5 \%)$ were treated with TMZ for two years. Their median time-to-progression was 28 months (95\% CI $=5.0-28.0)$, and they had an increased survival probability at three years compared to other patients (log-rank test $\left.\chi^{2}(1, \mathrm{~N}=56)=19.2, \mathrm{p}<0.0001\right)$.

Conclusions. There may be an advantage for a longer duration of TMZ therapy among patients with GBM, but the sample size was too small for generalization. A multicenter prospective study is needed to identify optimal duration of TMZ therapy.

\section{Kans J Med 2019;12(3):65-69.}

\section{INTRODUCTION}

Background and Rationale. Brain tumors account for $2 \%$ of all cancers, but their share of cancer morbidity and mortality remains disproportionate. ${ }^{1}$ Gliomas account for more than $80 \%$ of primary central nervous system (CNS) malignancies. Malignant, high-grade gliomas are grade III (which include anaplastic astrocytomas, anaplastic oligodendrogliomas, and anaplastic oligoastrocytoma), and grade IV glioblastomas (GBM). Most malignant gliomas are GBMs.

The Central Brain Tumor Registry of the United States (CBTRUS) reported 311,202 new cases of malignant and benign brain and CNS tumors from 2005 to 2009 . $^{2}$ The average annual age-adjusted incidence rates for GBM were 2.53 and 3.98 per 100,000 person-years for females and males, respectively. The male to female incidence ratio was 1.58 , whereas the white to black ratio was 2.06. GBMs constituted $15.8 \%$ of all reported tumors and $54 \%$ of all gliomas. The most prevalent locations of all brain tumors were the frontal lobe (25.6\%) and the temporal lobe (19.6\%). The CBTRUS also reported the mortality rate from malignant brain and CNS tumors was 3.48 for females and 5.23 for males per 100,000 person-years.

The five-year survival rate for GBM is $4.7 \% .^{3}$ Despite a multidisciplinary and aggressive treatment approach, GBM continues to have the lowest five-year survival rates among all human cancers. ${ }^{4}$ The median survival time from diagnosis rarely exceeds 12 months, and may even be shorter. ${ }^{5,6}$ Patients who survive for more than 36 months are considered long-term survivors.

Treatment of GBM is challenging due to resistant tumor cells, the innate fragility of the brain, and the inability of most chemotherapeutic agents to cross the blood-brain barrier. Historically, the standard therapy consisted of surgical resection followed by radiotherapy. Early clinical trials of the effectiveness of radiotherapy versus radiotherapy plus concomitant chemotherapy displayed no statistically significant survival benefits of chemotherapy. ${ }^{7}$ However, more recent clinical trials and long-term analyses have identified a specific chemotherapeutic agent, temozolomide (TMZ), as conferring a statistically significant survival benefit when given concurrently with radiation therapy (as opposed to radiotherapy alone). ${ }^{8,9}$ Following the Phase III trial by Stupp et al., ${ }^{8}$ the current standard of care treatment for newly diagnosed GBM after gross-total resection is based on concurrent radiation therapy with concomitant TMZ followed by six cycles of adjuvant TMZ, known as the Stupp protocol.

Adverse effects of TMZ include myelosuppression and significant thrombocytopenia. However, studies have suggested no significant increase in TMZ toxicity with prolonged treatment. ${ }^{10,11}$ For elderly patients diagnosed with GBM, six cycles may be sufficient. ${ }^{12}$ The clinical trials that originally established the benefit of TMZ utilized a six cycle (six-month) period of treatment. ${ }^{8}$ Adjuvant TMZ therapy for longer than six cycles may yield superior progression-free results and overall survival without TMZ toxicity. ${ }^{12-16}$ The optimal duration of therapy with TMZ remains unknown.

The objective of this study was to evaluate the survival benefit, if any, of a longer treatment duration with TMZ using an intent-totreat period of two years, given that all the other treatment guidelines have been followed. 


\section{METHODS}

The study protocol was approved by the Institutional Review Board at the University of Kansas School of Medicine-Wichita. The study design consisted of a retrospective chart review of patients with GBM who were treated at a cancer referral center in the Midwest, with an intent-to-treat protocol under which patients were to be treated for two years of adjuvant therapy with TMZ. The results were compared to historical controls and data from previous clinical trials, where the patients were treated for up to one year. Electronic medical charts were the source of the study data. The registry was searched using keywords: brain neoplasm, GBM, and glioblastoma. The results were filtered according to diagnosis date so that only those treated between January 1, 2002 and December 31, 2011 were selected if inclusion and exclusion criteria were met.

Patients were selected according to the following inclusion criteria: 18 years or older, with a GBM diagnosis, received TMZ as first-line chemotherapy, with the intent to treat for two years. All eligible patients had surgery with the intent of gross total resection, followed by imaging assessment within 24 to 48 hours of surgery, and concurrent radiation therapy and TMZ within two to four weeks of diagnosis, depending on the need for rehabilitation. The continuous variables that were abstracted included age, time to progression, and time to death. The categorical variables collected included Karnofsky Performance Score (KPS), gender, surgical intervention, external pathology report verification, and the use and type of seizure medications. Survival was calculated as the time from surgical diagnosis until death or end of the study period, whichever came first. The date of death was registered and verified through hospital records or the obituary regardless of the cause. The date of diagnosis, date of progression, and date of death were compared to calculate time interval variables. The outcome of interest was survival.

Anticonvulsant therapy was collected as a possible prognostic factor. Information on patients who presented with seizures and who were on antiepileptic therapy was collected. For patients who did not require it therapeutically, antiepileptic therapy also could be started as prophylaxis after brain surgery for about three months. There had been some concern that the concomitant use of anti-epileptic medication like hepatic metabolism inducers, which includes many first generation antiepileptic medications, may increase the myelotoxicity of chemotherapeutic agents. However, the 2010 European Society for Medical Oncology clinical practice guidelines do not agree, specifically as it pertains to temozolomide ${ }^{13}$ Regardless, the potential effect modification of treatment efficacy among the treatment cohort was evaluated by the use of antiepileptic medication.

Potential confounders included type of surgical resection, inaccurate pathology report, and demographics. Patients with missing data about progression time and survival time were censored in the analysis. Censoring was assumed to be independent of likely adverse reaction to treatment, patient performance status, or treatment effectiveness. Misclassification bias was addressed by recording whether the pathology report was reviewed at an external pathology laboratory.
KANSAS JOURNAL of MEDICINE GLIOBLASTOMA AND INCREASED SURVIVAL continued.

Statistical Methods. All data were collected in Microsoft Excel (version 2007) and analyzed using Statistical Analytics Software (SAS) version 9.3. Analysis was conducted to determine if there was a relationship between the duration of treatment and long-term survival of patients with GBMs. All tests were evaluated at a $\mathrm{p}=0.05$ or less level of significance. The overall survival from GBM was defined as time from surgical resection until death from all possible causes. Patients for whom no definite event status (i.e., death) was ascertainable, like those lost to follow-up, were considered censored. Patients who were alive also were considered censored and accounted for in evaluating patient overall survival duration.

The Kaplan-Meier method with log-rank test was used to estimate the overall survival (OS) probability distribution of patients after treatment. To adjust for potential confounding of overall survival by patient demographics and clinical characteristics, the Cox Proportional Hazards (PH) regression model was used to evaluate the relationship between survival and those covariates. Covariates that had significant influence in determining survival from GBM were assessed using the stepwise method of variable selection. Progression-free survival (PFS) also was evaluated. The event of interest in this respect was progression or recurrence of disease within two years of start of treatment. Patients who were lost to follow-up before their disease progressed were censored. The Kaplan-Meier method with log-rank test was used to estimate the progression-free survival distribution of patients after treatment.

\section{RESULTS}

The initial search yielded 211 patients, of which 109 had GBM. Since TMZ was not first-line treatment until around 2005, all patients before that period who were treated with other agents were excluded, resulting in a total of 66 patients. Of the 66 patients, there were 25 females and 41 males. The mean age at diagnosis was 60 years (standard deviation of 12.5). A total of 56 patients were included in the analysis because of the lack of survival data on 10 patients. Seven patients (12.5\%) completed two full years of TMZ (Table 1). The median age was 62 years for the whole group (56 patients) and 64 for the group treated with two years of TMZ. The median Karnofsky's performance score (KPS) was 80 for both groups.

All the patients in the study were on an intent-to-treat protocol for a period of two years, but the median survival time was 13 months (SE $=0.068)$. The two-year survival probability was $28.3 \%(\mathrm{SE}=0.064)$ and the three-year survival probability was $17.8 \%(\mathrm{SE}=0.059)$. At five years, the computed survival probability was $4 \%(\mathrm{SE}=0.041)$. 
KANSAS JOURNAL of MEDICINE GLIOBLASTOMA AND INCREASED SURVIVAL

continued.

Table 1. Patient characteristics.

\begin{tabular}{|c|c|c|}
\hline & $\begin{array}{c}2 \text { Years Treatment, } \\
\text { n }(\%)\end{array}$ & $\begin{array}{l}\text { Total, } \mathbf{n} \\
(\%)\end{array}$ \\
\hline \multicolumn{3}{|l|}{ Age } \\
\hline $20-44$ & $2(28.6)$ & $5(7.5)$ \\
\hline $45-54$ & & $13(19.7)$ \\
\hline $55-64$ & $2(28.6)$ & $23(34.9)$ \\
\hline $65-74$ & $2(28.6)$ & $18(27.3)$ \\
\hline$>74$ & $1(14.2)$ & $7(10.6)$ \\
\hline \multicolumn{3}{|l|}{ Gender } \\
\hline Male & $6(14.6)$ & $41(62.1)$ \\
\hline Female & $1(4.0)$ & $25(37.9)$ \\
\hline \multicolumn{3}{|l|}{ Tumor localization } \\
\hline \multicolumn{3}{|l|}{ Hemisphere $^{\mathrm{a}}$} \\
\hline Right & $5(71.4)$ & $37(58.7)$ \\
\hline Other & $2(28.6)$ & $26(41.3)$ \\
\hline \multicolumn{3}{|l|}{ Lobe $^{\mathrm{b}}$} \\
\hline Frontal & $4(57.2)$ & $26(39.4)$ \\
\hline Temporal & $1(14.2)$ & $21(31.8)$ \\
\hline Other & $2(28.6)$ & $19(28.8)$ \\
\hline \multicolumn{3}{|l|}{ Treatment } \\
\hline \multicolumn{3}{|l|}{ Tumor resection } \\
\hline Total & $5(71.4)$ & $41(63.1)$ \\
\hline Sub-total & $2(28.6)$ & $20(30.8)$ \\
\hline Biopsy & & $4(6.1)$ \\
\hline Radiotherapy & $7(100.0)$ & $66(100.0)$ \\
\hline Progression & $3(42.9)$ & $40(67.8)$ \\
\hline External pathology review & $5(71.4)$ & $32(51.6)$ \\
\hline Antiepileptic medications & $5(71.4)$ & $50(75.8)$ \\
\hline Enzyme inducers & $3(60.0)$ & $26(52)$ \\
\hline Non enzyme inducers & $2(40.0)$ & $24(48)$ \\
\hline
\end{tabular}

aThe data on three were missing.

${ }^{\text {b}}$ For tumors that involved more than one lobe, it was reported according to the lobe that was most affected. In addition, there was one tentorial, one intraventricular, one insular, one over the corpus callosum and one with multiple masses on the left. They were all grouped with occipital and parietal masses under "other".

The median time to progression for the study group was eight months. Patients who were treated with two years of TMZ (Figure 1) had a statistically significant increase in the likelihood of survival compared to those with less than two years of treatment (log-rank test $\left.\chi^{2}(1, \mathrm{~N}=56)=19.2, \mathrm{p}<0.0001\right)$. Their median time to progression was 28 months. Survival was increased significantly for those who had a good KPS compared to fair KPS (log-rank test $\chi^{2}(1, N=$ $56)=8.5, \mathrm{p}=0.0034$; Figure 2). Survival was also higher among those who had a tumor located in the right cerebral hemisphere, compared to those with a tumor in other locations $\left(\log\right.$-rank test $\chi^{2}(1, N=56)=$ $5.5, \mathrm{p}=0.018$; Figure 3$)$. Patients who had successful gross resection had a better chance of survival than those who had a biopsy, but only a marginal increase in overall survival compared to those who had a sub-total resection $\left(\right.$ log-rank test $\left.\chi^{2}(2, \mathrm{~N}=56)=3.8, \mathrm{p}=0.15\right)$. Those who progressed had decreased survival compared to those who did not have progression in their disease. Gender, location, pathology review, and the use and type of anti-convulsive medications appeared to have no effect on patient survival. Younger patients aged 19 - 44 (age groups: 19 - 44, 45 - 54, 55 - 64, 65 - 74, and older than 74 years) had a marginal increase in survival, although this was not statistically significant (log-rank test $\chi^{2}(4, N=56)=5.27, p=0.26$; Figure 4).

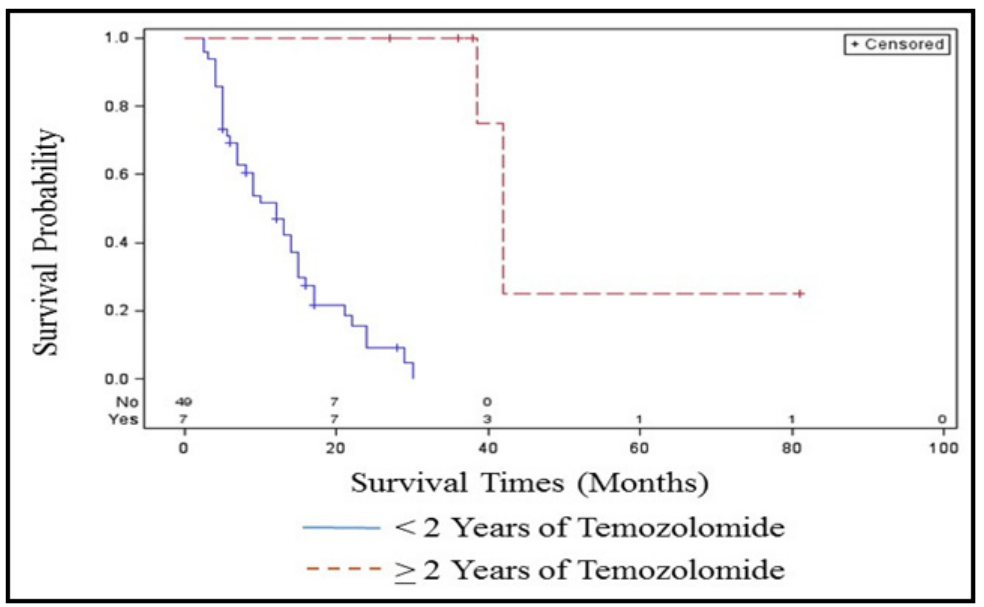

Figure l. Overall survival with number of subjects at risk by treatment duration of two years or less of Temozolomide.

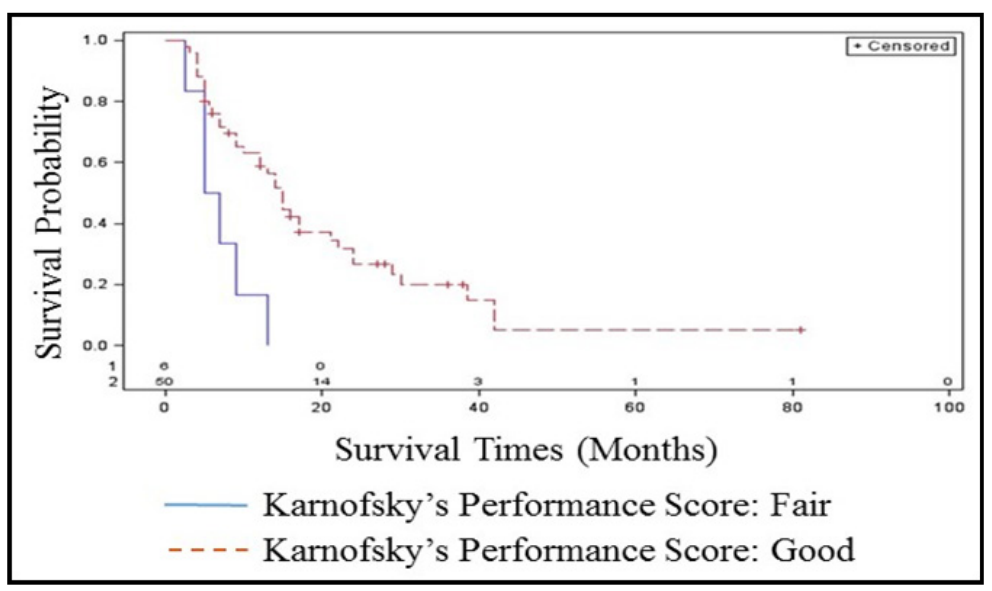

Figure 2. Overall survival with number of subjects at risk by Karnofsky's Performance Score.

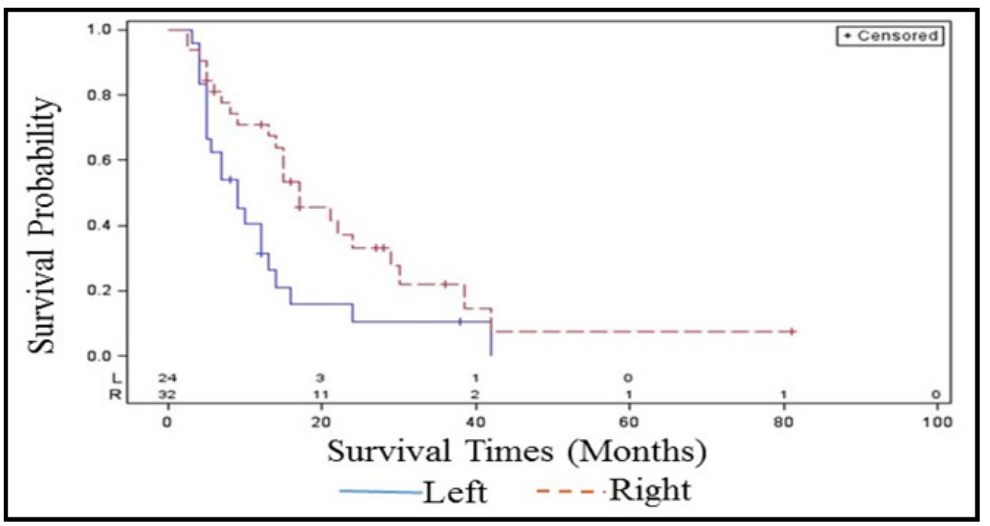

Figure 3. Overall survival with number of subjects at risk by brain hemisphere location of the tumor. 


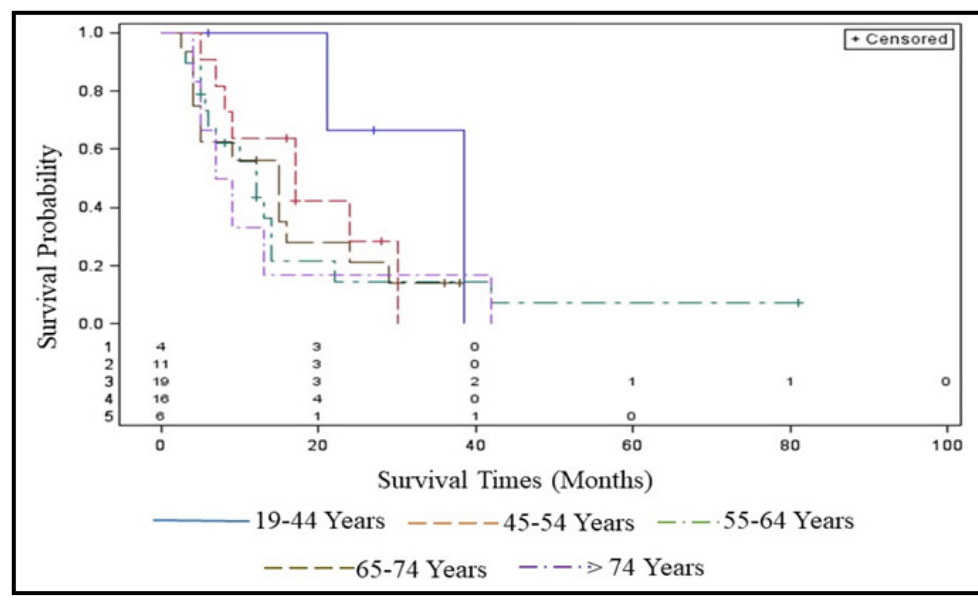

Figure 4. Overall survival with number of subjects at risk by age group.

After univariate adjustment, good KPS and the location of the tumor in the right hemisphere showed significant effect on survival (Table 2). After adjusting for other potential predictors in a multivariable analysis, only patients with tumors sited in the right side of the brain continued to show any significant benefit with treatment (HR $=3.077,95 \% \mathrm{CI}=1.159-8.172$; Table 2). Multivariable analysis also indicated that the risk of death due to GBM was reduced for persons aged 45 to 54 years compared to those aged 75 years or older (HR $=0.161,95 \% \mathrm{CI}=0.029$ - 0.883; Table 2). The two-year treatment group analysis suggested a persistent survival advantage, even after adjustment, but due to the small sample size, the precision of the effect size was not reliable.

Table 2. Multivariable adjusted analysis.

\begin{tabular}{|c|c|c|c|c|}
\hline & \multicolumn{2}{|c|}{ Univariate } & \multicolumn{2}{|c|}{ Multivariate } \\
\hline & $\begin{array}{c}\text { Hazard } \\
\text { Ratio }\end{array}$ & $95 \% \mathrm{CI}$ & $\begin{array}{c}\text { Hazard } \\
\text { Ratio }\end{array}$ & $95 \% \mathrm{CI}$ \\
\hline $\begin{array}{l}\text { Age-groups } \\
\text { (vs. }>74 \text { ) }\end{array}$ & & & & \\
\hline $20-44$ & 0.301 & $0.060-1.521$ & 0.407 & $0.038-4.366$ \\
\hline $45-54$ & 0.566 & $0.190-1.683$ & 0.161 & $0.029-0.883$ \\
\hline $55-64$ & 0.853 & $0.330-2.206$ & 0.573 & $0.123-2.676$ \\
\hline $65-74$ & 0.788 & $0.290-2.136$ & 0.529 & $0.099-2.822$ \\
\hline Female (vs Male) & 1.346 & $0.724-2.502$ & 0.910 & $0.376-2.201$ \\
\hline KPS fair (vs good) & 3.491 & $1.394-8.746$ & 2.970 & $0.570-15.485$ \\
\hline $\begin{array}{l}\text { Central } \\
\text { (vs other region) }\end{array}$ & 0.514 & $0.234-1.132$ & 0.412 & $0.153-1.109$ \\
\hline $\begin{array}{l}\text { Excision: } \\
\text { sub-total (vs total) }\end{array}$ & 1.521 & $0.805-2.873$ & 1.168 & $0.531-1.109$ \\
\hline $\begin{array}{l}\text { Excision: biopsy } \\
\text { (vs total) }\end{array}$ & 2.380 & $0.818-6.928$ & 0.717 & $0.099-5.917$ \\
\hline $\begin{array}{l}\text { EIAC }^{*} \\
\text { (vs non-EIAC) }\end{array}$ & 0.716 & $0.373-1.376$ & .844 & $0.356-2.001$ \\
\hline $\begin{array}{l}\text { Pathology review: } \\
\text { no (vs yes) }\end{array}$ & 0.884 & $0.482-1.623$ & 0.990 & $0.416-2.353$ \\
\hline $\begin{array}{l}\text { Left hemisphere } \\
\text { (vs right) }\end{array}$ & 1.989 & $1.086-3.643$ & 3.077 & $1.159-8.172$ \\
\hline $\begin{array}{l}\text { Location: frontal } \\
\text { (vs temporal) }\end{array}$ & 1.208 & $0.575-2.538$ & 1.589 & $0.628-4.022$ \\
\hline $\begin{array}{l}\text { Location: other } \\
\text { (vs temporal) }\end{array}$ & 1.304 & $0.589-2.886$ & 0.970 & $0.247-3.809$ \\
\hline
\end{tabular}

*Enzyme inducing anticonvulsant.
KANSAS JOURNAL of MEDIC INE

GLIOBLASTOMA AND INCREASED SURVIVAL

continued.

\section{DISCUSSION}

This study suggested an increase in overall survival for patients who successfully completed two years of TMZ therapy. As previously mentioned, the optimal duration of therapy using TMZ has not been determined and there are limited data available in the literature. ${ }^{14}$ In fact, upon extensive literature review, only six small studies have evaluated the efficacy of TMZ for an extended period of treatment. ${ }^{10-12,14,17,18}$ In a 2007 study published by Hau et al., ${ }^{10}$ patients with GBM receiving first-line TMZ for a median 13 cycles had a median PFS of 14 months. Seiz et al..$^{19}$ found a statistically significant correlation between the number of adjuvant TMZ cycles and both PFS and OS in 59 patients treated with more than six cycles of TMZ. Other subsequent studies noted a statistically significant increase in PFS and OS for patients treated with more than six cycles of adjuvant monthly TMZ..$^{12,14,20}$ As the side effects of TMZ generally are welltolerated, it seemed reasonable to evaluate the outcome of patients who were treated for a longer period of time..$^{10,11}$ Although studies have evaluated the long-term efficacy of TMZ for glioblastoma, this is one of the first studies that examined treatment duration of two years with TMZ.

According to population-based studies, the median age at diagnosis of GBM is 64 years. $^{2}$ This is similar to our study where both patients who completed two years of TMZ and the sample as a whole had a median age above 60 years. Age subgroup analysis suggested survival advantage only for those 45 to 54 years compared to those older than 74 years. This is likely related to sample sizes in each subgroup, which may not be large enough to make predictive conclusions of differential treatment benefits in these subgroups. Also, our patient population exhibited a relatively high KPS, which has been associated with longer survival. In fact, all patients who completed two years of TMZ had a good KPS score; however, the multivariate adjustment suggested no effect of KPS on survival (Table 2). These results provide an important insight in potential treatment considerations among patients with GBM and it may be of particular interest to evaluate in larger clinical trials whether the longer treatment duration should be assessed in all GBM patients or only those younger than 70 years old with good KPS.

In a 2007 analysis of 55 long-term survivors of GBM by Krex et al., ${ }^{4}$ several clinical and molecular features were associated with longterm survival. However, this study failed to include details regarding pathology review and treatment regimen. Inadvertent inclusion of patients with lower grade gliomas, for instance, could have skewed their results toward a more favorable outcome. In contrast, in our series, all patients except four had evidence of secondary confirmation of the GBM diagnosis, and more than half had the results externally reviewed at another center (Table 1). 


\section{KANSAS JOURNAL of MEDICINE}

\section{GLIOBLASTOMA AND INCREASED SURVIVAL} continued.

Our study was consistent with the CBTRUS data regarding male to female ratio (1.64 vs 1.58, respectively), median age, and the most prevalent locations being frontal and temporal lobes. ${ }^{2}$ However, contrary to the previously published data, the current study suggested that good KPS and total resection were not associated with increased survival compared to fair KPS and sub-total resection, after adjusting for other potential covariates. In our series, we also observed a longer survival for those patients diagnosed with right-sided GBM than those with left-sided GBM. Whether this is simply an artifact of this patient cohort is unknown. A potential explanation could be, however, that since the left hemisphere is commonly the dominant hemisphere and the location of the language center, left-sided GBM may result in worse outcomes because of loss of essential functions.

Limitations. There are several potential limitations to this study. First, the sample size was not adequate to allow for generalization. However, most studies involving GBM and TMZ have relatively small sample sizes. A prospective, multicenter study that can include a larger sample size might evaluate the question of duration of TMZ therapy better. Secondly, this study was a retrospective chart review with some missing data that may have introduced bias to the results. For instance, one long-term survivor (81 months) in the successfully treated group had no external pathology review. Although a pathology review also may have confirmed the GBM diagnosis, there were isolated case reports about very long-term survival with verified GBM, and one patient still alive after 21 years. ${ }^{20}$ Additionally, DNA molecular data for the patients were not available for this study. Therefore, we were not able to compare survival according to O6-methylguanine-DNA methyltransferase (MGMT) promoter methylation status, which is predictive of the response to TMZ and a favorable prognostic factor in GBM..$^{15,18}$ More recently, over-expression of the Deltrex-1 (DTXI) gene was correlated with increased cell migration and invasion, whereas over-expression of SLC7A7 predicts poor PFS and OS in patients with GBM. ${ }^{16,21}$ Additionally, some technology was not available for this study. For instance, newer studies about surgical resection and survival in GBM have evaluated a new technology consistent with fluorescence-guided resection. Complete surgical resection of the enhancing tumor on early MRI and no fluorescent residual tissue can have longer OS compared to those with residual fluorescent tissue. ${ }^{22}$

In conclusion, this study suggested that there may be a survival advantage with a longer duration of TMZ therapy in patients with GBM, regardless of age, KPS score, or type of surgical resection. In the last five years, there has been an increase in data, which suggested a benefit of this strategy on PFS and OS. Our study was one of the first studies to evaluate treatment duration of two years with TMZ in patients with GBM. While we considered the sample size to be too small for generalization, larger prospective studies might evaluate the question of duration of TMZ therapy better, particularly if both clinical and basic science data are paired.

\section{REFERENCES}

${ }^{1}$ Jacobs JF, Idema AJ, Bol KF, et al. Regulatory T cells and the PD-Ll/PD-1 pathway mediate immune suppression in malignant human brain tumors. Neuro Oncol 2009; 11(4):394-402. PMID: 19028999.

2 Dolecek TA, Propp JM, Stroup NE, Kruchko C. CBTRUS statistical report: Primary brain and central nervous system tumors diagnosed in the United States in 2005-2009. Neuro Oncol 2012; 14(Suppl 5):vl-v49. PMID: 23095881.

${ }^{3}$ Ries LAG, Eisner MP, Kosary CL, et al. SEER Cancer Statistics Review, 1975-2001, National Cancer Institute. Bethesda, MD. Available at: http:// seer.cancer.gov/csr/1975_2001/,2004. Accessed July 26, 2018.

${ }^{4}$ Krex D, Klink B, Hartmann C, et al. Long-term survival with glioblastoma multiforme. Brain 2007; 130(10):2596-2606. PMID: 17785346.

${ }^{5}$ Smith JS, Jenkins RB. Genetic alterations in adult diffuse glioma: Occurrence, significance, and prognostic implications. Front Biosci 2000; 5:D213-31. PMID: 10702383.

${ }^{6}$ Ohgaki H, Dessen P, Jourde B, et al. Genetic pathways to glioblastoma: A population-based study. Cancer Res 2004; 64(19):6892-6899. PMID: 15466178.

Stewart LA. Chemotherapy in adult high-grade glioma: A systematic review and meta-analysis of individual patient data from 12 randomised trials. Lancet 2002; 359:1011-1018. PMID: 11937180.

${ }^{8}$ Stupp R, Mason WP, van den Bent MJ, et al. Radiotherapy plus concomitant and adjuvant temozolomide for glioblastoma. N Engl J Med 2005; 352(10):987-996. PMID: 15758009.

${ }^{9}$ Erpolat OP, Akmansu M, Goksel F, Bora H, Yaman E, Buyukberber S. Outcome of newly diagnosed glioblastoma patients treated by radiotherapy plus concomitant and adjuvant temozolomide: A long-term analysis. Tumori 2009; 95(2):191-197. PMID: 19579865.

${ }^{10}$ Hau P, Koch D, Hundsberger T, et al. Safety and feasibility of long-term temozolomide treatment in patients with high-grade glioma. Neurology 2007; 68(9):688-690. PMID: 17325277.

${ }^{11}$ Khasraw M, Bell D, Wheeler H. Long-term use of temozolomide: Could you use temozolomide safely for life in gliomas? J Clin Neurosci 2009; 16(6):854-855. PMID: 19303779.

12 Minniti G, De Sanctis V, Muni R, et al. Radiotherapy plus concomitant and adjuvant temozolomide for glioblastoma in elderly patients. J Neurooncol 2008; 88(1):97-103. PMID: 18250965.

${ }^{13}$ Stupp R, Tonn JC, Brada M, Pentheroudakis G, ESMO Guidelines Working Group. High-grade malignant glioma: ESMO Clinical Practice Guidelines for diagnosis, treatment and follow-up. Ann Oncol 2010; 21(Suppl 5):vl90-193. PMID: 20555079.

${ }^{14}$ Holdhoff M, Grossman SA. Controversies in the adjuvant therapy of highgrade gliomas. Oncologist 2011; 16(3):351-358. PMID: 21339260.

${ }^{15}$ Zhang K, Wang XQ, Zhou B, Zhang L. The prognostic value of MGMT promoter methylation in Glioblastoma multiforme: A meta-analysis. Fam Cancer 2013; 12(3):449-458. PMID: 23397067.

${ }^{16}$ Huber RM, Rajski M, Sivasankaran B, Moncayo G, Hemmings BA, Merlo A. Deltex-l activates mitotic signaling and proliferation and increases the clonogenic and invasive potential of U373 and LN18 glioblastoma cells and correlates with patient survival. PLoS One 2013; 8(2):e57793. PMID: 23451269.

17 Barbagallo GM, Paratore S, Caltabiano R, et al. Long-term therapy with temozolomide is a feasible option for newly diagnosed glioblastoma: A single-institution experience with as many as 101 temozolomide cycles. Neurosurg Focus 2014; 37(6):E4. PMID: 25434389.

${ }_{18}$ Hegi ME, Diserens AC, Gorlia T, et al. MGMT gene silencing and benefit from temozolomide in glioblastoma. N Engl J Med 2005; 352(10):9971003. PMID: 15758010.

19 Seiz M, Krafft U, Freyschlag CF, et al. Long-term administration of temozolomide in patients with glioblastoma multiforme: Experience of a single institution. J Cancer Res Clin Oncol 2010; 136(11):1691-1695. PMID: 20177703.

${ }^{20}$ Fukushima S, Narita Y, Miyakita Y, et al. A case of more than 20 years survival with glioblastoma, and development of cavernous angioma as a delayed complication of radiotherapy. Neuropath 2013; 33(5):576-581. PMID: 23406431.

${ }^{21}$ Fan S, Meng D, Xu T, et al. Overexpression of SLC7A7 predicts poor progression-free and overall survival in patients with glioblastoma. Med Oncol 2013;30(1):384. PMID: 23408368.

${ }_{22}$ Aldave G, Tejada S, Pay E, et al. Prognostic value of residual fluorescent tissue in glioblastoma patients after gross total resection in 5-aminolevulinic acid-guided surgery. Neurosurgery 2013; 72(6):915-920. PMID: 23685503. 